\title{
Acknowledgement to Reviewers of Resources in 2019
}

\author{
Resources Editorial Office \\ MDPI, St. Alban-Anlage 66, 4052 Basel, Switzerland \\ Published: 17 January 2020
}

The editorial team greatly appreciates the reviewers who have dedicated their considerable time and expertise to the journal's rigorous editorial process over the past 12 months, regardless of whether the papers are finally published or not. In 2019, a total of 173 papers were published in the journal, with a median time to first decision of 15 days and a median time from submission to publication of 41 days. The editors would like to express their sincere gratitude to the following reviewers for their generous contribution in 2019:

\begin{tabular}{ll} 
Abbas, Azhar & Banias, George \\
Aceleanu, Mirela & Bányai, Tamás \\
Ackah, Ishmael & Baranyai, Nóra \\
Adam, Paul & Barbosa, Belem \\
Adham, Ammar & Bargallo, Ramon \\
Aldieri, Luigi & Barral Silva, María Teresa \\
Alexander, Malov & Barrile, Vincenzo \\
Alfano, Vincenzo & Bartocci, Pietro \\
Alfonso, Leonardo & Bartoli, Mattia \\
Amirkhani, Masoume & Bartzas, Georgios \\
Amitrano, Cristina Caterina & Bascompta, Marc \\
An, Chunjiang & Bastante-Ceca, Maria Jose \\
Anand, Adarsh & Benedict, Barry \\
Anezakis, Vardis-Dimitris & Beňová, Mariana \\
Annema, Jan Anne & Bentes, Isabel \\
Antczak, Elzbieta & Bethurem, Matthew \\
Aolin, Alina & Bhat, Sartaj Ahmad \\
Archetti, Francesco & Bhattarai, Arjun \\
Aricò, Costanza & Bickford, Nate \\
Aseeva, Anna & Biswas, Bhaba \\
Atanasoae, Pavel & Blanco, Julien \\
Augustine, Swinburne A.J. & Bogacki, Jan \\
Azevedo, Leonardo & Bolognesi, Thomas \\
Baena, Francisco Manuel & Borden, Kira A. \\
Bahers, Jean-Baptiste & Borge-Diez, David \\
Bahmanteymouri, Elham & Borrelli, Luigi \\
Baimel, Dmitry & Bostan, Ionel \\
Bajić, Dragoljub & Bostenaru Dan, Maria \\
Baldacchini, Chiara & Bournaris, Thomas \\
Balomenos, Efthymios & Brauer, Dave \\
\hline
\end{tabular}


Brillard, Alain

Brodny, Jarosław

Brzychczy, Edyta

$\mathrm{Bu}$, Siqi

Burduhos, Bogdan-Gabriel

Burja, Jaka

Burlea-Schiopoiu, Adriana

Bustillo-Lecompte, Ciro

Busu, Cristian

Busu, Mihail

Bywater-Reyes, Sharon

Caetano, Nídia

Cai, Jialiang

Calin, Adrian Cantemir

Caloiero, Tommaso

Caranta, Roberto

Carcavilla, Luis

Carral Vilariño, Emilio V.

Caruzzo, Amaury

Caterina, Caviglia

Cerbu, Camelia

Ceresia, Francesco

Chakraborty, Abhik

Chambel, Luis

Chang, Ya-Ju

Chapman, Andrew John

Chen, Han-Shen

Chen, Yi-Tui

Chendev, Yury

Cherepovitsyn, Alexey

Christoforidis, Georgios C.

Chylińska, Dagmara

Cieślińska, Barbara

Cifuentes, Hector

Coates, Ken

Coman, Mihaela Denisa

Comanescu, Laura

Cook, Steve

Cooney, Rosie

Corbí, Hugo

Corrêa Magalhães Filho, Fernando Jorge

Cristian, Dumitru

Csomós, György
Cumming, Douglas

Cvetković, Marko

Dąbrowska, Dominika

Dąbski, Maciej

D'Adamo, Idiano

Dahl, Martin

Dalaibuyan, Byambajav

De Donatis, Mauro

De Jong, Wil

De Koning, Arjan

De Los Ríos-Berjillos, Araceli

Defilippis, Paolo

Demartini, Paola

Dembińska, Izabela

Denić-Jukić, Vesna

Dewitt, Jessica

Di Noia, Luigi Pio

Diaz-Balteiro, Luis

Dipu, Arnoldus Lambertus

Dombek, Grzegorz

Domenech, Teresa

Döring, Ralf

Doroudiani, Saeed

Drąg, Paweł

Dragovic, Besim

Drejerska, Nina

Drielsma, Johannes

Drosos, Vasileios C.

Du, Jia-Chong

Dumitriu, Dan

Duque, Carlos

Durán-Barroso, Pablo

Đurin, Bojan

Eberle, Paul

Eden, Susanna

Edwards, Keith

Emmer, Adam

Enserink, Bert

Erkut, Burak

Esteban, M. Dolores

Evangelou, Eleftherios

Falcone, Pasquale Marcello

Fan, Huan-Jung 


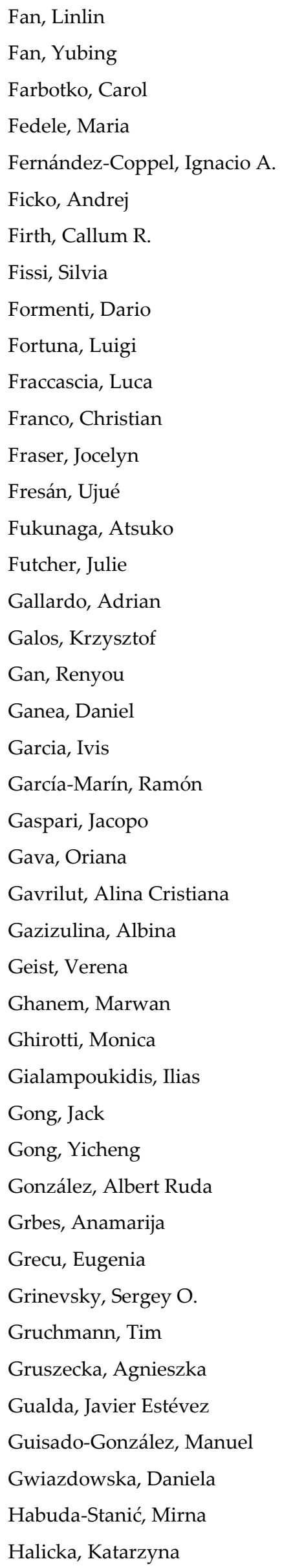

Hameed, Maysoun

Hannouf, Marwa

Harbulakova, Vlasta

Harrington, Julie

Hasager, Charlotte Bay

Hawken, Scott

Heo, Wookjae

Hettiarachchi, Hiroshan

Hitch, Michael

Hodgkinson, Jane

Hoeksema, Bert

Hoffmann, Markus M.

Hoghooghi, Nahal

Hong, Boe-Shong

Hou, Jingwei

HSIEH, Chi-Ming

$\mathrm{Hu}$, Jin-Li

Huang, Kuo-Tsang

Husgafvel, Roope

Hussain, Suhail S.M.

Inkinen, Tommi

Interis, Matthew

Ionescu, Gabriela

Irimias, Anna

Irlbacher-Fox, Stephanie

Jacka, Jerry

Jackson, Sara

Jahromi, Hossein

Jakić, Miće

Jantrania, Anish

Japelj, Anže

Ji, Jian

Jorge Evangelista, Ana Catarina

Joshi, Sanjeev

Juhna, Talis

Juliano, Claudia Clelia Assunta

Jurado, Enrique

Jursová, Simona

Kalligeros, Stamatis Spyridon

Kang, Kai

Karbowski, Adam

Karczmarczyk, Agnieszka

Kardos, Mihaela 
Kelly, Geoff

Khan, Tehmina

Kidd, Petra

Kim, Doyoon

Kim, Kee Dae

Kim, Myung Kyo

Kim, Seoyong

Kim, Seung-Nam

Kirchner, Stefan

Klemes, Jiri Jaromir

Kolokoltsov, Vassili

Konstantakopoulou, Foteini

Kopylov, Yuri

Korf, Nathalie

Kosinski, Eryk

Koulinas, Georgios K.

Kozak, Miłosław

Kozhevnikov, Mikhail

Kraal, Diane

Kumar BATAR, Amit

Kuo, Shu-lung

Kupidura, Przemysław

Lai, Sabrina

Latosińska, Jolanta

Laubie, Baptiste

Lawhon, Lydia A.

Ledda, Antonio

Lee, Jejung

Lee, Sungju

Lekavičius, Vidas

Lekunberri, Edorta Carrascal

Lesniewska, Feja

Lettner, Miriam

Lezzi, Greta Enrica

Li, Biao

Li, Frank

Li, Yang

Lin, Hsin-Tien

Lin, Jia

Lin, Teng-Chiu

Liobikienè, Genovaitè

Liolios, Konstantinos A.

Liu, Shuangzhe
Liu, Wan-Yu

Logan, Lauren H.

Longo, Rocco

Lopez, Felix A.

Loubota Panzou, Grace Jopaul

Loures, Luís

Lovarelli, Daniela

Łuczak, Sergiusz

Lujan-Alvarez, Concepcion

Lukač, Niko

Madadian, Edris

Madonia, Paolo

Magaril, Elena

Maineult, Alexis

Maksymiuk, Gabriela

Maletskyi, Zakhar

Mancini, Lucia

Manera, Carles

Manno, Giorgio

Mannu, Alberto

Manzano, Osmel

Marek, Michal V.

Marino, Davide

Markides, Christos N.

Marković, Tamara

Markowicz-Piasecka, Magdalena

Marta-Costa, Ana Alexandra

Martí-Ballester, Carmen Pilar

Martinat, Stanislav

Martins, Bruno

Matsui, Kenichi

Matykiewicz, Danuta

Mavragani, Amaryllis

McGrattan, Kevin B.

Medyna, Galyna

Megerle, Heidi

Melelli, Laura

Menor-Campos, Antonio

Mentel, Grzegorz

Meyer-Kohlstock, Daniel

Miah, Mohammad Islam

Miglietta, Pier Paolo

Migoń, Piotr 
Milichovský, František

Milosavljević, Milutin M.

Mion, Giorgio

Moiseyev, Alexander

Moliner, Cristina

Momete, Daniela

Moreno Liso, Maria Lourdes

Moretti, Laura

Morrone, Domenico

Mourato, Sandra

Mozumder, Mohammad

Mrzljak, Vedran

Muhammad-Sukki, Firdaus

Müller, Julian Marius

Mulloy, Barbara

Murakami, Shinsuke

Muresan, Iulia C.

Muro Jr, Aldo

Mutu, Margaret Shirley

Myszograj, Sylwia

Nadolny, Zbigniew

Nafees, Lubna

Nafi, Amir

Nara, Satya

Naranjo, José Manuel

Narduzzi, Luca

Nastasi, Benedetto

Nazarko, Lukasz

Ndi, George

Neto, Susana

Nguyen, Khoi

Niemann, Jorg

Nižić, Marinela Krstinić

Northey, Stephen

Novo, Luís

Nunes, Leonel

Nunes, Sara

Núñez, Remigio Paradelo

Núñez-Cacho Utrilla, Pedro

Nutile, Samuel

Oda, Takuya

Okpara, Uche

O'Leary, Simon
Oncioiu, Ionica

Ondrejka Harbuláková, Vlasta

Onishi, Viviani

Ortiz, Julian

Orynycz, Olga

Orzolek, Michael D.

Pach, Maciej

Paci, Chris

Pagano, Alessandro

Palus, Hubert

Panepinto, Deborah

Panico, Antonio

Pappila, Minna

Pásková, Martina

Paudyal, Ramesh

Pauliuk, Stefan

Paunovic, Ivan

Paz, Igor

Peach, James

Pearce, Prafula

Pereira, Adelino J.C.

Pereira, Maria Teresa

Petr, Novák

Petrikova, Ivica

Petrisor, Alexandru-Ionut

Petrosanu, Dana-Mihaela

Petrosino, Paola

Petti, Luiga

Pierluigi, Brandolini

Pietrucha-Urbanik, Katarzyna

Pires Manso, Jose' Ramos

Pirjan, Alexandru

Policht-Latawiec, Agnieszka

Pomares, Juan Carlos

Poponi, Stefano

Privitera, Donatella

Proença De Oliveira, Rodrigo

Puig-Bargués, Jaume

Pulka, Jakub

Rada, Elena

Radianti, Jaziar

Rahbar, Kiyarash

Rahman, Md Mofijur 
Rajulapati, Chandra Rupa

Rak, Janusz

Rassõlkin, Anton

Ratner, Albert

Ravina, Marco

Raźniak, Piotr

Reddy, Yenumula B.

Renau-Pruñonosa, Arianna

Rendon, Olivia

Ricart, Sandra

Rico Amorós, Antonio

ROBERT, Hassink

Robustelli, Gaetano

Rocha, Ada Margarida Correia Nunes Da

Rodrigo-Comino, Jesus

Rodrigues, Abel

Rodríguez-Monroy, Carlos

Roehrich, Jens

Rosskopf, Carmen Maria

Roumpos, Christos

Roy, Partho Sarothi

Ruban, Dmitry

Ruckstuhl, Katharina

Rudzińska, Magdalena

Ruiz-Real, Jose Luis

Rusu, Teodor

Rutkowski, Jacqueline Elizabeth

Ryen, Erinn

Sadoi, Yuri

Sahoo, Kamalakanta

Sajdak, Marcin

Salinas, Jennifer

Samper, María Dolores

Sansò, Paolo

Sasaki, Daisuke

Sattary, Sattar

Sava, Gabriela

Schiavon, Marco

Schmidt, Mario

Schneider, Daniel Rolph

Schneider, Petra

Schopfel, Joachim

Seifan, Mostafa
Seják, Josef

Selvaratnam, Thinesh

Šerić, Ljiljana

Settembre Blundo, Davide

Sharifi, Ayyoob

Sheehy, Benedict

Sidor, Csaba

Sierra Fernández, José María

Siksnelyte, Indre

Skoulikaris, Charalampos

Skripnuk, Djamilia

Smith, James

Smułek, Wojciech

Soares Mota, Luís Cândido

Socoliuc, Marian

Şoimoșan, Teodora Melania

Solar, Hector

Someus, Edward

Song, Xuehang

Song, Yungoo

Sousa, Hélder S.

Spilka, Monika

Stasis, Antonios

Stauvermann, Peter Josef

Stepanova, Nadezhda Yuilevna

Strba, Lubomir

Štverková, Hana

Sulek, Rastislav

Sun, Zhi

Suppa, Domenico

Szabó-Takács, Beáta

Szczepaniak, Włodzimierz

Szczepankiewicz, Elżbieta

Szilas, Kristoffer

Szmelter-Jarosz, Agnieszka

Szymańska-Pulikowska, Agata

Tadić, Lidija

Taghizadeh, Ruhollah

Taylor, Smith

Tcvetkov, Pavel

Teah, Heng-Yi

Thomas, Terry

Thorleifson, Harvey 


Todeschini, Sara
Toma, Lucian
Topor, Marcel
Toscani, Philipp
Tran, Carolyn
Tricarico, Carla
Tsai, Wen-Hsien
Tsvetkov, Nikolai
Tun, Maw Maw
Tutak, Magdalena
Umbleja, Kadri
Uprety, Yadav
Vaidya, Ashma
Valeev, Dmitry
Valentin, Herbei Mihai
Van Der Voorn, Tom
Van Zelst, Sebastiaan
Varbanov, Petar
Vasconcelos, Helena Cristina
Vasconi, Mauro
Vaverkova, Magdalena
Verlicchi, Paola
Vithana, Chamindra
Vlad, Ciprian
Vokoun, Melinda
Von Jouanne, Annette
Wandosell, Gonzalo
Wang, Hua
Wang, Ruofan

Wasiak, Andrzej

Wei, Xuan

Wigger, Henning

Wise, Nicholas

Witczak, Joanna

Wohner, Bernhard

Wrona, Paweł

Wulf, Christina

Wurl, Jobst

Xavier, António M.S.

Xenidis, Yiannis

Xue, Bing

Yang, Weixin

Yang, Xue-Ming

Yang, Yang

Yee, Susan

Yen, Tian-Ming

Yilmaz, Erol

Yoon, Young-Man

Zamfir, Rares Halbac-Cotoara

Zangeneh-Nejad, Farzad

Zawieja, Iwona

Zema, Demetrio

Zglobicki, Wojciech

Zhang, Pengfei

Zimon, Dominik

Zsembeli, József

Zubelzu Minguez, Sergio

(C) 2020 by the author. Licensee MDPI, Basel, Switzerland. This article is an open access article distributed under the terms and conditions of the Creative Commons Attribution (CC BY) license (http://creativecommons.org/licenses/by/4.0/). 